\title{
AURORAL SIGNATURES OF SATURN'S MAGNETOSPHERIC DYNAMICS
}

\author{
S. Badman*
}

\begin{abstract}
Saturn's aurorae have been observed at UV, IR, and visible wavelengths by space- and Earth-based instruments. The aurorae are usually brighter on the dawn side, indicating an influence by the solar wind via enhanced flow shear at the dawn flank. However, the intensity at all local times is also modulated independently in the north and south by the so-called planetary period oscillations. Diffuse features equatorward of Saturn's main emission have been related to scattering of particles in quasi-steady (nightside secondary arc) or transient (dayside patches) processes. Signatures of magnetic reconnection have been identified as arcs and spots on both the dayside and nightside, with particularly broad and intense nightside features occurring in response to solar wind compressions. These auroral features and the underlying magnetospheric dynamics will be discussed in relation to concurrent radio emissions such as low frequency extensions of the Saturn Kilometric Radiation, and $1 \mathrm{~h}$ pulsations in auroral hiss.
\end{abstract}

${ }^{*}$ Lancaster University, Lancaster, UK 
